\title{
A!
}

This is an electronic reprint of the original article.

This reprint may differ from the original in pagination and typographic detail.

Todoshchenko, I.; Savin, A.; Haataja, M.; Kaikkonen, J. P.; Hakonen, P. J.

\section{Quartz tuning fork as a probe of surface oscillations}

Published in:

Applied Physics Letters

DOI:

$10.1063 / 1.4976093$

Published: 13/02/2017

Document Version

Publisher's PDF, also known as Version of record

Please cite the original version:

Todoshchenko, I., Savin, A., Haataja, M., Kaikkonen, J. P., \& Hakonen, P. J. (2017). Quartz tuning fork as a probe of surface oscillations. Applied Physics Letters, 110(7), 1-5. [071601]. https://doi.org/10.1063/1.4976093

This material is protected by copyright and other intellectual property rights, and duplication or sale of all or part of any of the repository collections is not permitted, except that material may be duplicated by you for your research use or educational purposes in electronic or print form. You must obtain permission for any other use. Electronic or print copies may not be offered, whether for sale or otherwise to anyone who is not an authorised user. 


\section{Quartz tuning fork as a probe of surface oscillations}

I. Todoshchenko, A. Savin, M. Haataja, J.-P. Kaikkonen, and P. J. Hakonen

Citation: Appl. Phys. Lett. 110, 071601 (2017); doi: 10.1063/1.4976093

View online: http://dx.doi.org/10.1063/1.4976093

View Table of Contents: http://aip.scitation.org/toc/apl/110/7

Published by the American Institute of Physics

\section{Articles you may be interested in}

Electrically driven and electrically tunable quantum light sources

Appl. Phys. Lett. 110, 071102071102 (2017); 10.1063/1.4976197

Tamper indicating gold nanocup plasmonic films

Appl. Phys. Lett. 110, 071101071101 (2017); 10.1063/1.4975936

Precision phase estimation based on weak-value amplification

Appl. Phys. Lett. 110, 071105071105 (2017); 10.1063/1.4976312

Pyroelectric response in crystalline hafnium zirconium oxide $(\mathrm{Hf1}-\mathrm{xZrxO} 2)$ thin films

Appl. Phys. Lett. 110, 072901072901 (2017); 10.1063/1.4976519

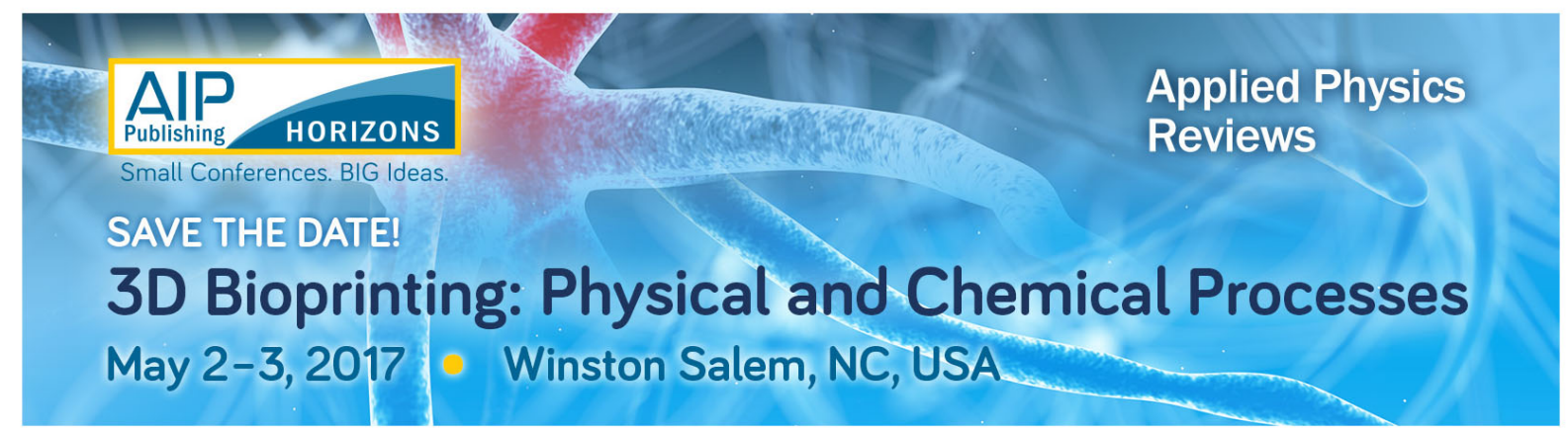




\title{
Quartz tuning fork as a probe of surface oscillations
}

\author{
I. Todoshchenko, ${ }^{\text {a) }}$ A. Savin, M. Haataja, J.-P. Kaikkonen, and P. J. Hakonen \\ Low Temperature Laboratory, Department of Applied Physics, Aalto University, Espoo 00076, Finland
}

(Received 2 December 2016; accepted 29 January 2017; published online 14 February 2017)

\begin{abstract}
Quartz tuning forks are high-quality mechanical oscillators widely used in low temperature physics as viscometers, thermometers, and pressure sensors. We demonstrate that a fork placed in liquid helium near the surface of solid helium is very sensitive to the oscillations of the solid-liquid interface. We developed a double-resonance read-out technique, which allowed us to detect oscillations of the surface with an accuracy of $1 \AA$ in 10 s. Using this technique, we have investigated crystallization waves in ${ }^{4} \mathrm{He}$ down to $10 \mathrm{mK}$. In contrast to previous studies of crystallization waves, our measurement scheme has very low dissipation, on the order of $20 \mathrm{pW}$, which allows us to carry out experiments even at sub-mK temperatures. We propose to use this scheme in the search for crystallization waves in ${ }^{3} \mathrm{He}$, which exist only at temperatures well below $0.5 \mathrm{mK}$. The suggested technique can also be used for accurate displacement detection in a large variety of systems. Published by AIP Publishing. [http://dx.doi.org/10.1063/1.4976093]
\end{abstract}

Interfaces in quantum fluids and solids display a variety of physical phenomena. In addition to usual capillarygravity waves, they are mobile enough to support also phase waves, like crystallization waves ${ }^{1}$ or massless phase waves $^{2}$ on the A-B interface of ${ }^{3} \mathrm{He}$. Besides the fact that these phase waves are very interesting and unusual on their own, they present a unique tool to investigate surface bound states. Surface quasiparticles contribute directly to the surface tension as well as to the dissipation of the surface waves, so that they can be detected by measuring the dispersion relation of the waves.

Usually, the contribution of surface excitations to the dissipation of the surface waves is masked by the interaction with particles in bulk phases. However, at low temperatures, the contribution from the bulk particles decreases as $T^{4}$ or even faster. ${ }^{3}$ Due to their $2 \mathrm{D}$ nature, surface excitations experience slower decay upon cooling, and at a low enough temperature, they provide the largest contribution to the dissipation of the waves. Helium is the only substance that remains liquid at ultralow temperatures and thus allows us to investigate surface waves and various surface bound states.

Manninen et $a l^{3,4}$ have performed pioneering experiments on capillary-gravity waves on the free surface of liquid ${ }^{3} \mathrm{He}$ well below the superfluid transition temperature of 1 $\mathrm{mK}$. Their measurements suggest that at temperatures below $0.2 \mathrm{mK}$ the damping of the capillary-gravity waves is no longer determined by the bulk quasiparticles, the density of which vanishes exponentially with decreasing temperature. ${ }^{3,4}$ Interestingly, different modes of the surface waves had different temperature dependence of dissipation, which may indicate wave-number dependent interaction with the surface states.

Crystallization waves in ${ }^{4} \mathrm{He}$ were predicted by Andreev and Parshin ${ }^{1}$ in 1978 and discovered two years later by Keshishev et al. at temperatures below $0.5 \mathrm{~K} .{ }^{5}$ By measuring the frequency of crystallization waves at crystal surfaces of different orientations, Rolley et al. have observed, as

${ }^{\text {a)} E l e c t r o n i c ~ m a i l: ~ i g o r . t o d o s h c h e n k o @ a a l t o . f i ~}$ predicted by Landau, ${ }^{6}$ a singularity in the surface tension at the orientation of the basal facet. ${ }^{7,8}$

In ${ }^{3} \mathrm{He}$, an interfacial wave between solid and superfluid phases is an even more intriguing object to study because it has to accommodate to the magnetically ordered phase of the solid and the $p$-wave-paired order parameter of ${ }^{3} \mathrm{He}$ superfluid. Due to the magnetically ordered solid, the crystallization wave is accompanied by spin supercurrents, which contribute to the inertia of this wave along with the regular mass flow, and in high enough magnetic fields the whole inertial mass of the wave is of a magnetic origin. However, due to the entropy related to magnetic degrees of freedom, crystallization waves in ${ }^{3} \mathrm{He}$ are strongly damped and can be observed only at temperatures well below $0.5 \mathrm{mK} .{ }^{9,10}$ This sub-mK temperature requirement sets strong limitations for the excitation of the waves because the electrical capacitors utilized to generate the waves may produce significant heat load to the helium sample.

In this letter, we suggest an extremely sensitive and low dissipation scheme for detection of surface waves. We have tested this scheme by observing crystallization waves in ${ }^{4} \mathrm{He}$ down to oscillation amplitudes of few nanometers. The obtained results show that the developed technique can be used to investigate surface waves in the sub-millikelvin range. We also discuss the possible use of the quartz tuning fork as an accurate displacement detector in other systems.

Excitation of surface waves at low temperatures is a very difficult experimental task. Generally, one needs to oscillate pressure at/near the surface. Moreover, the oscillating pressure should be applied locally because a spatially uniform pressure variation will oscillate the surface as a whole $(q=0)$, instead of producing waves on it. However, the speed of sound $c=366 \mathrm{~m} / \mathrm{s}$ (Ref. 12) in liquid helium at the melting pressure is much faster than the speed of surface waves, which means that the needed pressure variation cannot be applied via liquid by any mechanical transducer. Instead, electrical capacitors partly immersed in helium can be used to create local pressure variation. Due to its electrical polarizability, helium acquires additional energy in the 
electric field. The electrical energy is equivalent to additional pressure

$$
\delta p=\varepsilon_{0}(\varepsilon-1) E^{2} / 2 .
$$

According to the Clausius-Mossotti relation, the permittivity $\varepsilon$ relates to the polarizability $\alpha$ by: $\varepsilon=\left(1+\frac{8 \pi}{3} \frac{\alpha}{v}\right) /$ $\left(1-\frac{4 \pi}{3} \frac{\alpha}{v}\right) \approx 1+4 \pi \frac{\alpha}{v}$, where $v$ is the molar volume. Among all atoms, the helium atom has the smallest polarizability, $\alpha_{H e}=0.1232 \mathrm{~cm}^{3} / \mathrm{mol}$, which is three times smaller than that of hydrogen. In addition, liquid helium is quite a rarefied medium with a molar volume of about $30 \mathrm{~cm}^{3} / \mathrm{mol}$ at $p=25 \mathrm{bar}$, almost twice that of water. These result in the very small value of $(\varepsilon-1)$ for condensed helium phases (0.054 for liquid ${ }^{4} \mathrm{He}$ and 0.052 for liquid ${ }^{3} \mathrm{He}$ at zero bar) and, correspondingly, in very low electrostatic pressure $\delta p$ from Eq. (1). To exemplify, an electrical field of $2 \mathrm{MV} / \mathrm{m}$ is needed to lift the liquid-vapour helium interface on a capacitor by $1 \mathrm{~mm}$. To decrease the voltage needed for such a high electric field, one can decrease the spacing $d$ in the capacitor. However, in this way the volume of the capacitor also decreases, which leads to a reduction in the relative share of the electric energy. Practical values of the spacing are thus set by the above limitations to the range $20-100 \mu \mathrm{m}$, and the corresponding voltages are a few hundred of volts.

In their experiments on crystallization waves, Rolley et al. utilized an interdigital capacitor on borosilicate glass with a periodicity of $80 \mu \mathrm{m} .^{7,8}$ They measured the dielectric losses in the substrate of the capacitor to be about $30 \mu \mathrm{W}$ at typical drives (170 V peak-to-peak @ $1 \mathrm{kHz})$, and such a high heat leak limited the temperature of their experiment to $40 \mathrm{mK}$. To reduce dielectric losses, we made our capacitor using a double winding of $60 \mu \mathrm{m}$ thin superconducting wire on a copper holder. Eddy current heating due to the displacement currents is minimized in this design because currents in neighboring wires flow in opposite directions. The amount of dielectric was minimized by choosing wires with a thin $(5 \mu \mathrm{m})$ insulation layer and using thin cigarette paper and very diluted GE-varnish. The height and the width of the capacitor were also kept small with $H=1.5 \mathrm{~mm}$ and $W=4 \mathrm{~mm}$, respectively, thus reducing the volume of dielectric material and the induced losses in proportion.
The solid-liquid interface of helium is even more difficult to drive because solid helium wets solid surfaces very poorly. The capillary forces preventing the solid from filling the narrow gap of the large field at the capacitor can be estimated as follows. Gravity and electric field contribute to the pressure in the liquid as $p_{l}-p_{0}=-\rho_{l} g h+\varepsilon_{0}\left(\varepsilon_{l}-1\right) E^{2} / 2$, where $h$ is the vertical position of the surface with respect to the reference level. The pressure within the solid having a single curvature radius $R$ is larger than that in the liquid by the Laplace pressure $p_{s}=p_{l}+\alpha / R$ where $\alpha$ is the surface tension and $R \sim d$ reflects the size of the capacitor spacing.

The electric pressure needed to compensate for capillary forces is thus $\varepsilon_{0}\left(\varepsilon_{l}-1\right) E_{c}^{2} / 2=\left(\rho_{l} / \Delta \rho\right)(\alpha / R)$, and substituting values ${ }^{10,11}$ for ${ }^{4} \mathrm{He}, \alpha=1.7 \times 10^{-4} \mathrm{~J} / \mathrm{m}^{2}$ and $\rho_{l} / \Delta \rho \approx 10$ we obtain for the critical field $E_{c} \approx 10 \mathrm{MV} / \mathrm{m}$. Fig. 1(a) displays areas near the capacitor's neighbouring wires where the $E>E_{c}$ at different voltages $U$ applied across the two capacitor electrodes. One can see that at voltages $U<100 \mathrm{~V}$, the gap with the high field is still too small to overcome the capillary forces, and first at $U \sim 200 \mathrm{~V}$ applied to the capacitor the gap becomes of the order of $d$ to fit the solid. After the capillary forces have been overcome, solid fills the volume of the capacitor, and the level of solid decreases in the rest of the cell.

To measure a sub-micron change of the level of solid, we have introduced a double-resonance method with a quartz tuning fork as a sensitive element. Tuning forks are nowadays widely exploited in helium low temperature experiments as thermometers, viscometers, pressure sensors, turbulence detectors, etc. ${ }^{13,14}$ In our measurement scheme resembling the so-called near-field acoustic microscopy, ${ }^{15,16}$ an oscillating quartz tuning fork is placed in the vicinity of the surface of the solid. A change in the distance $z$ between the interface and the fork distorts the velocity field in the liquid around the oscillating fork and thus changes its effective hydrodynamic mass. This causes a change in the resonance frequency of the fork. As the quality factor of the fork at low temperatures may be as high as $10^{6}$, a very tiny detuning of the resonance frequency can be detected.

Our measurement scheme is shown in Fig. 1(b). A high AC voltage $U=U_{0} \sin \pi F t$ at a low frequency $F / 2=1 \ldots$ $50 \mathrm{~Hz}$ is applied to the capacitor, which drives the interface (a)

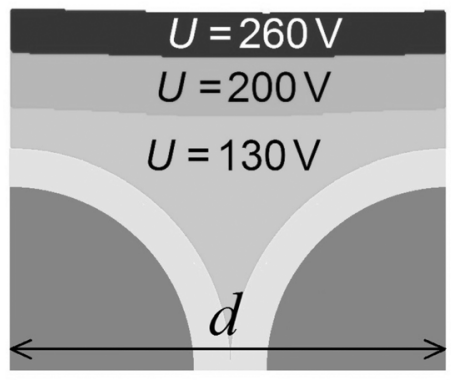

(b)

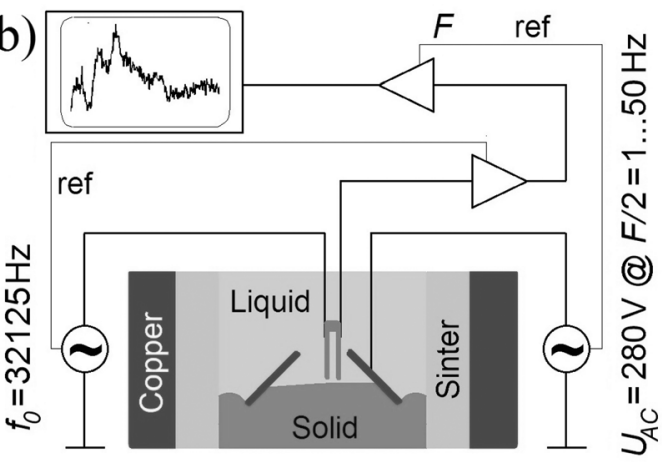

FIG. 1. (a) Calculated areas of high $(>10 \mathrm{MV} / \mathrm{m}$ ) field in the vicinity of two neighboring wires of the capacitor at different voltages. (b) Schematic illustration of the experimental cell and the read-out scheme using two lock-in amplifiers. The fork is placed in the liquid just above the surface of solid and driven at its resonance frequency $f_{0}=32125 \mathrm{~Hz}$. The fork current is measured by the first lock-in amplifier. High AC voltage applied to the capacitor at frequency $F / 2$ causes the surface of solid to oscillate at frequency $F$, which leads to a periodical detuning of the fork. The detuning results in the oscillation of the quadrature current through the fork, which is measured by another lock-in amplifier at frequency $F=2 \ldots 100 \mathrm{~Hz}$. 
at the frequency $F$ because the electrostatic pressure is proportional to the square of the field. The fork is driven at frequency $f_{0}=32125 \mathrm{~Hz}$ close to (oscillating) resonance frequency $f_{\text {res }}(z)$ and the quadrature of the fork output current is demodulated using another lock-in detector synchronized with $U^{2}$ at frequency $F$. When $F$ coincides with the standing surface wave resonance frequency, a maximum appears in the quadrature oscillation amplitude, which is proportional to the amplitude of the surface oscillation.

The experimental cell is a vertical cylinder of $35 \mathrm{~mm}$ diameter directly joined to a heat exchanger on a copper nuclear demagnetization stage. ${ }^{17} \mathrm{~A}$ silver sinter for heat exchange is baked on copper plates in the form of stacked horizontal layers of $2 \mathrm{~mm}$ thickness with a $10 \mathrm{~mm}$ hole in the center. ${ }^{18}$ The semi-open helium resonator is made using four thin copper plates to separate the space. One of the plates holds a wire-wound capacitor to generate waves. Plates are $1.5 \mathrm{~mm}$ high and $5 \mathrm{~mm}$ wide each. All four plates are tilted by $45^{\circ}$ to compensate for the wetting angle of solid helium. ${ }^{19}$ The fork is placed at a distance of $0.5 \mathrm{~mm}$ from the center towards the middle of the wave generator. A schematic horizontal cross-section of the cell is shown in the inset of Fig. 3(b). The experiments were performed on a Bluefors LD-400 dilution refrigerator.

Crystals were grown using the so-called blocked capillary method ${ }^{20}$ in which the cell is pressurized at temperature $T \approx 2 \mathrm{~K}$ and then cooled down. The solid fraction at low temperature $x=V_{s} / V_{t o t}$ can be calculated from $x=\left[\rho_{l}(T)\right.$ $\left.-\rho_{l}(0)\right] /\left[\rho_{s}(0)-\rho_{l}(0)\right]$. In our experiments, the solid fraction $x=0.20$ was calculated to provide solid right below the fork, which corresponds to $\rho_{l}(T)=176.4 \mathrm{~kg} / \mathrm{m}^{3}$. ${ }^{11}$ We chose to pressurize the cell at $T=1.7 \mathrm{~K}$ where the pressure providing the needed density was calculated to be 27.18 bar. After cooling down below $200 \mathrm{mK}$, the fork became frozen in solid. Then, we carefully let some helium to release from the cell until the resonance re-appeared. Releasing helium was made very slowly in order not to melt too much solid because it was crucial for sensitive measurements that the fork was placed as close to the crystal surface as possible.

Since the velocity field around fork prongs decays very fast with the distance, the detuning of the fork caused by the perturbation of the velocity field also decreases fast with the distance from the solid. This is illustrated in Fig. 2(a) showing the calculated distribution of the velocity of the liquid around the fork at different distances $z$ from the solid, and in Fig. 2(b) showing the detuning $\Delta f_{0} \equiv f_{\text {res }}(z)-f_{0}$ of the fork as a function of the distance.
The calculations were done using the finite element method. The fork employed in our experiment had $2.45 \mathrm{~mm}$ long, $0.10 \mathrm{~mm}$ wide, and $0.24 \mathrm{~mm}$ thick prongs with a gap of $0.12 \mathrm{~mm}$ between the prongs. The simulation space was a $6 \mathrm{~mm} \times 6 \mathrm{~mm} \times 10 \mathrm{~mm}$ rectangular prism, which typically consisted of $\sim 4 \times 10^{5}$ tetrahedral domain elements. The domain elements were more highly concentrated near the fork and its tip, where changes in the velocity field are more precipitous. The fork was placed along the central $z$-axis, while the bottom $x y$-plane was made into a "solid" by applying a zero-flux von Neumann boundary condition (BC). The same BC was applied on the fork surfaces, except for the sides with their normal parallel to the prong's movement. These sides had the movement implemented by applying non-zero flux BCs, which increased closer to the tip according to the fork's velocity profile. The kinetic energy of the liquid was then calculated and the added hydrodynamical mass was obtained. The corresponding shift of the resonance frequency of the fork as a function of distance $z$ is shown in Fig. 2(b) together with the fitted empirical relation suggested by Callaghan et al. ${ }^{21}$ In the inset of Fig. 2(b), we plot the inverse sensitivity $d z / d \Delta f_{0}$ of the fork to a displacement of the crystal surface as a function of the distance $z$.

Fig. 3(a) displays the measured detuning of the fork due to a change in the ${ }^{4} \mathrm{He}$ solid-liquid interface position as a function of the DC voltage applied to the capacitor. As is seen from the figure, there is a threshold voltage of about $210 \mathrm{~V}$ above which the crystal starts filling the capacitor, and the level of the solid near the fork decreases. The value of the threshold is in good agreement with the estimations of the capillary forces and with the calculations of the electrical field shown in Fig. 1(a). At higher voltages, the detuning increases slowly because the field decays exponentially with the distance from the capacitor, and, correspondingly, the volume of the high field region increases only logarithmically with the voltage. The estimated volume of the capacitor is about $0.05 \mathrm{~mm}^{3}$, which corresponds to a $0.5 \mu \mathrm{m}$ change of the level of solid in the rest of the cell. The inverse sensitivity $d z / d \Delta f_{0}$ of the measurement scheme is thus $0.5 \mu \mathrm{m} / 20$ $\mathrm{mHz}=2.5 \times 10^{-8} \mathrm{~m} / \mathrm{mHz}$, from which we infer that the fork is about $0.2 \mathrm{~mm}$ above the level of the solid, according to the calculated values shown in the inset of Fig. 2(b).

The proximity of the solid surface results not only in the frequency shift but also in an additional dissipation due to the viscous drag of the liquid at the solid surface. In our case, the liquid was superfluid, but nevertheless remarkable (a)

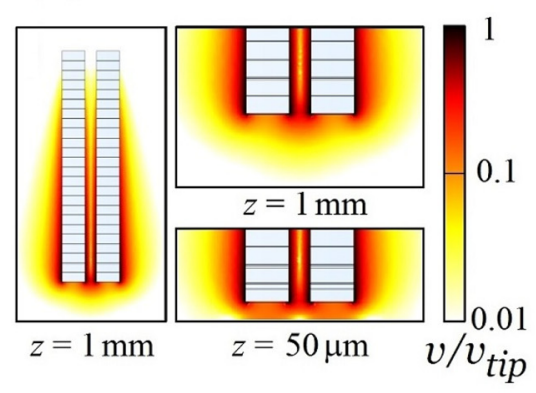

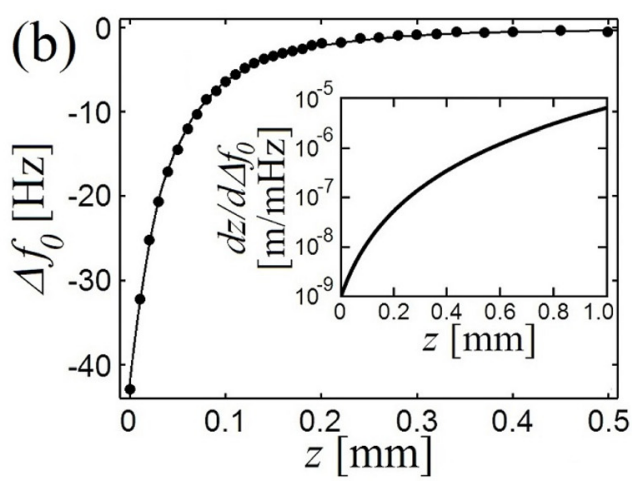

FIG. 2. (a) Calculated absolute velocities of the liquid in the central plane around oscillating fork placed at different distances $z$ from the solid wall. (b) Calculated detuning $\Delta f_{0} \equiv f_{\text {res }}(z)-f_{0}$ of the fork as a function of the distance $z$. Inset: inverse sensitivity of the measurement scheme to the change of the position of the interface. 
(a)

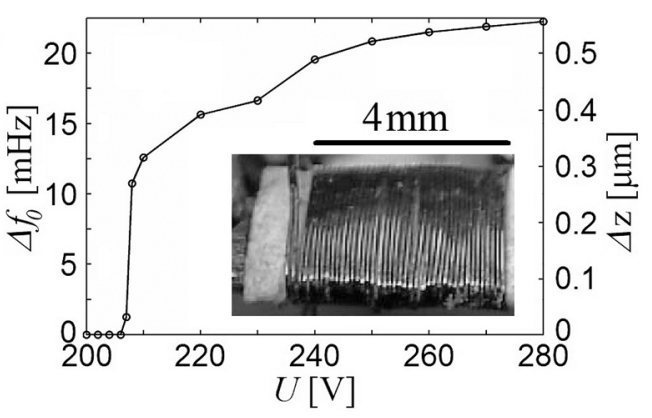

(b)

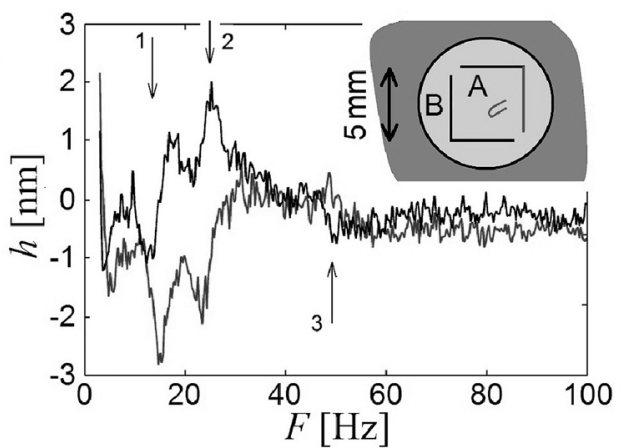

FIG. 3. (a) Change in the detuning of the fork as a function of the DC voltage applied to the capacitor. On the right, we present a displacement scale estimated from the volume of the capacitor divided by area of the crystal surface and the calculated detuning. Inset: photo of the capacitor. (b) Spectrum of crystallization waves in ${ }^{4} \mathrm{He}$. Light: in-phase response; dark: out-of-phase response. Numbers " 1, , " 2 ," and " 3 " mark the fundamental resonance and its harmonics, which involve both the inner and outer regions A and B, respectively. Inset: schematic view of the horizontal cross-section of the cell.

additional attenuation of the order of $1 \mathrm{~Hz}$ was observed. We attribute this damping to generation of the surface waves by the oscillating velocity-pressure field of the liquid.

The spectrum of crystallization waves in ${ }^{4} \mathrm{He}$ measured at $10 \mathrm{mK}$ at $U_{0}=280 \mathrm{~V}$ AC-drive is shown in Fig. 3(b). The integration time of the second lock-in amplifier measuring detuning of the fork at the frequency of the $U_{A C}^{2}$ was $10 \mathrm{~s}$. The first two resonances at 13 and $22 \mathrm{~Hz}$ correspond to the fundamental resonance and its first harmonic across the whole crystal-liquid interface (our wave generator excites the interface on both sides and the inner and outer regions are strongly coupled on the liquid side). The third peak at $\sim 48 \mathrm{~Hz}$ is assigned to a higher harmonic, which is weakly coupled to the fork owing to its asymmetric mode shape with respect to the oscillation detector. One can also notice that the efficiency of a generation of the waves decreases with increasing frequency $F$, probably due to the limited growth rate of ${ }^{4} \mathrm{He}$ crystals. ${ }^{22-24}$ As seen from Fig. 3(b), the resolution of this measurement scheme is about $1 \AA$. Equally good resolution has been reached earlier in measurements on crystal growth ${ }^{25}$ with a much more complicated optical setup.

We note that the physical principles of the displacement detection in our method are quite general and are not restricted to ultra low temperatures. Indeed, the velocity field of any fluid or gas around the fork will be perturbed by the proximity of any boundary. The resolution of the method depends on the quality factor $Q$ of the fork in the given medium, on the density $\rho$ of medium, and on the distance from the fork to the measured surface. To exemplify, we consider air at normal conditions where the quality factor of our fork is 7 times lower than in the crystallization wave experiment. The density of air at normal temperature and pressure (NTP) is 200 times smaller than that of liquid ${ }^{4} \mathrm{He}$ at melting pressure, which means that the frequency shift, and, consequently, the sensitivity are also 200 lower. On the other hand, the position of the fork at NTP conditions can be easily controlled: placing the fork at a distance of few microns to the surface allows increasing the sensitivity by a factor of 20, see inset of Fig. 2(b). Taking into account a lower quality factor, we thus estimate that the resolution of our scheme in an air at normal conditions will be about $70 \AA$. In other gases or liquids, the resolution scales as $\rho / Q$, which is proportional to the square root of the inverse kinematic viscosity of the medium.

In order to test the heating by the capacitor, we made a separate cooldown in which we condensed ${ }^{3} \mathrm{He}$ in the same cell. At the melting pressure and at the lowest temperature of $0.39 \mathrm{mK}=0.16 T_{c}$, we have measured the dissipation produced by the driving capacitor at different frequencies. The dissipation was found to depend on the frequency as $f^{2}$, indicating that the main heat source is dielectric losses in the isolating materials of the capacitor. Eddy current heating would be proportional to $f^{4}$ since the electromagnetic induction $\mathscr{E}$ in the copper plate is proportional to the time derivative of the displacement current in the capacitor, $\mathscr{E} \propto \dot{I}=(2 \pi f)^{2} C U$, and the dissipation is proportional to $\mathscr{E}^{2}$. The heat released at the maximum frequency of $50 \mathrm{~Hz}$ and $U_{0}=280 \mathrm{~V}$ amplitude warmed the helium sample from $0.39 \mathrm{mK}$ to $0.40 \mathrm{mK}$ in about $1 \mathrm{~min}$. Estimating the heat capacity of our $1 \mathrm{~mol}$ helium sample at this temperature as $C \sim R \exp (-\Delta / T)=5 \times 10^{-5} \mathrm{~J} / \mathrm{K}$ we find a heat leak of $P \sim 20 \mathrm{pW}$. This very small value of the heat leak should be compared with the heat leak from the interdigital capacitor in other works on crystallization waves in ${ }^{4} \mathrm{He},{ }^{7,8}$ where, extrapolated to our frequencies and voltage, it would be 70 $\mathrm{nW}$, i.e., more than three orders of magnitude larger. The reason for such a strong reduction of dissipation was, we believe, the use of a very tiny $6 \mathrm{~mm}^{2}$ wound capacitor with a minimum possible amount of dielectric material.

To summarize, we have developed a very sensitive technique for measuring oscillations of the solid-liquid interface of helium (crystallization waves). This double-resonance technique has been demonstrated to detect amplitudes of surface oscillations as small as $1 \AA$. Minimizing the dielectric losses in the capacitor resulted in a very small power dissipation of $20 \mathrm{pW}$. Such an ultra-low-dissipation technique allows experiments with solid-liquid interface of helium-3 well below $0.5 \mathrm{mK}$, where crystallization waves have been predicted to appear. The technique can also be used effectively to probe waves at the free surface of superfluid or at the interface between two different superfluid phases.

This work was supported by the Academy of Finland (Grant No. 284594, LTQ CoE), by the European Research Council (Grant No. 670743), and by Vilho, Yrjö and Kalle Väisälä Foundation of the Finnish Academy of Science and Letters. This research made use of the OtaNano-Low Temperature Laboratory infrastructure of Aalto University that is part of the European Microkelvin Platform. 
${ }^{1}$ A. F. Andreev and A. Ya. Parshin, Sov. Phys. - JETP 48, 763 (1978).

${ }^{2}$ I. Todoshchenko, Phys. Rev. B 93, 134509 (2016).

${ }^{3}$ M. S. Manninen, J. Rysti, I. Todoshchenko, and J. Tuoriniemi, Phys. Rev. B 90, 224502 (2014).

${ }^{4}$ M. S. Manninen, A. Ranni, J. Rysti, I. Todoshchenko, and J. Tuoriniemi, J. Low Temp. Phys. 183, 399 (2016).

${ }^{5}$ K. O. Keshishev, A. Ya. Parshin, and A. V. Babkin, JETP Lett. 30, 56 (1980).

${ }^{6}$ L. D. Landau, Collected Papers (Pergamon Press, Oxford, 1971).

${ }^{7}$ E. Rolley, S. Balibar, and F. Graner, Phys. Rev. E 49, 1500 (1994).

${ }^{8}$ E. Rolley, C. Guthmann, E. Shevalier, and S. Balibar, J. Low Temp. Phys. 99, 851 (1995).

${ }^{9}$ A. F. Andreev, Czech. J. Phys. 46, 3043 (1996).

${ }^{10}$ S. Balibar, H. Alles, and A. Ya. Parshin, Rev. Mod. Phys. 77, 317 (2005).

${ }^{11}$ E. R. Grilly, J. Low Temp. Phys. 4, 615 (1971).

${ }^{12}$ B. M. Abraham, Y. Eckstein, J. B. Ketterson, M. Kuchnir, and P. R. Roach, Phys. Rev. A 1, 250 (1970).

${ }^{13}$ M. Blazková, M. Clovecko, V. B. Eltsov, E. Gazo, R. de Graaf, J. J. Hosio, M. Krusius, D. Schmoranzer, W. Schoepe, L. Skrbek et al., J. Low Temp. Phys. 150, 525 (2008).
${ }^{14}$ S. L. Ahlstrom, D. I. Bradley, S. N. Fisher, A. M. Guénault, E. A. Guise, R. P. Haley, S. Holt, O. Kolosov, P. V. E. McClintock, G. R. Pickett et al., J. Low Temp. Phys. 175, 725 (2014).

${ }^{15}$ P. Günther, U. Ch. Fischer, and K. Dransfeld, Appl. Phys. B 48, 89 (1989).

${ }^{16}$ R. Steinke, M. Hoffmann, M. Böhmisch, J. Eisenmenger, K. Dransfeld, and P. Leiderer, Appl. Phys. A 64, 19 (1996).

${ }^{17}$ I. Todoshchenko, J.-P. Kaikkonen, R. Blaauwgeers, P. J. Hakonen, and A. Savin, Rev. Sci. Instrum. 85, 085106 (2014).

${ }^{18}$ R. H. Salmelin, J. M. Kyynäräinen, M. P. Berglundn, and J. P. Pekola, J. Low Temp. Phys. 76, 83 (1989).

${ }^{19}$ S. Balibar, C. Guthmann, and E. Rolley, J. Phys. I France 3, 1475 (1993).

${ }^{20}$ C. A. Swenson, Phys. Rev. 89, 538 (1953).

${ }^{21}$ F. D. Callaghan, X. Yu, and C. J. Mellor, Appl. Phys. Lett. 81, 916 (2002).

${ }^{22} \mathrm{P}$. Nozierès, in Solids far from equilibrium, edited by C. Godrèche (Cambridge University Press, 1989).

${ }^{23} \mathrm{~V}$. Tsepelin, H. Alles, A. Babkin, R. Jochemsen, A. Ya. Parshin, and I. Todoshchenko, J. Low Temp. Phys. 129, 489 (2002).

${ }^{24}$ J. P. Ruutu, P. J. Hakonen, J. S. Penttilä, A. V. Babkin, J. P. Saramäki, and E. B. Sonin, Phys. Rev. Lett. 77, 2514 (1996).

${ }^{25}$ J. P. Ruutu, P. J. Hakonen, A. V. Babkin, A. Ya. Parshin, and G. Tvalashvili, J. Low Temp. Phys. 112, 117 (1998). 